\title{
Examining Mathematics Anxiety of Undergraduates Using a Brain-Based Measurement, EEG
}

\author{
Anderson Norton ${ }^{*}$, Youngmin Seok ${ }^{2}$, Sangsook Choi-Koh ${ }^{2 \#}$ \\ ${ }^{1}$ Department of Mathematics, Virginia Tech, Blacksburg, VA, USA \\ ${ }^{2}$ Department of Mathematics Education, Dankook University, Yongin, South Korea \\ Email: "sangch2906@gmail.com
}

How to cite this paper: Norton, A., Seok, Y. and Choi-Koh, S. (2019) Examining Mathematics Anxiety of Undergraduates Using a Brain-Based Measurement, EEG Journal of Behavioral and Brain Science, 9, 195-209.

https://doi.org/10.4236/jbbs.2019.95017

Received: April 25, 2019

Accepted: May 24, 2019

Published: May 27, 2019

Copyright () 2019 by author(s) and Scientific Research Publishing Inc. This work is licensed under the Creative Commons Attribution International License (CC BY 4.0).

http://creativecommons.org/licenses/by/4.0/

\begin{abstract}
This paper reports on an investigation of mathematics anxiety (MA) among 40 Korean undergraduate students, using cognitive neuroscience. In Spring 2015, we collected data on correct response rates and reaction times from computer-based activities related to quadratic functions. We also measured brain response through event related potentials (ERP). Results demonstrate that students with higher mathematics anxiety (HMA) took more time than students with lower mathematics anxiety (LMA), both in translating equations to graphs and in translating graphs to equations. Moreover, based on analysis of ERP, brain waves of the HMA group recorded higher amplitude. In specific, both groups showed higher amplitude in translation from graphs to equation than vice versa. Higher amplitudes indicate greater demands on working memory, which we discuss in the concluding section, especially with regard to MA.
\end{abstract}

\section{Keywords}

Algebra, Electroencephalography, Event-Related Potentials, Function, Mathematics Anxiety, Neuroimaging, Undergraduate

\section{Introduction}

The topic of mathematics anxiety (MA) in mathematics education has been of great interest since [1] first developed the Mathematics Anxiety Rating Scale (MARS), which made it possible to diagnose students' MA. In Korea, issues in the affective domain are receiving more and more attention, particularly in rela-

*First author. 
tion to building up positive dispositions toward mathematics. The mathematics achievement of Korean students is top-ranked, but scores for positive affective domains in mathematics-such as interest and confidence-are not higher than the international average [2]. This result is not surprising because mathematics education in Korea has focused on computational and procedural approaches for rapid problem-solving responses, rather than creative approaches to problem solving or the application of mathematics to meaningful situations [3]. Sustained negative emotion toward mathematics is a prevalent phenomenon in Korean schools [4].

MA has been difficult to analyze, but the advancement of tools in brain science technology has made it possible to measure MA and its effects using electroencephalography (EEG). EEG is the measurement of activity among populations of neurons firing across the cerebral cortex, and it has been used to monitor different states of alertness or consciousness [5] [6]. We conducted a study to find features of MA via brain-based measurement, in order to understand what happens in students' brains while solving mathematics problems. Results have implications for alleviating MA in mathematics education.

In order to investigate the relationship MA and EEG measures, we chose to study students' problem solving in the context of functions, as represented by equations and graphs. Functions can be quite challenging, even for college students [7] [8]. We studied task responses from two groups of college students: students with lower MA (LMA) and students with higher MA (HMA). Within that setting, we posed the following research questions:

1) Was there a difference between the percentages of correct responses (PCR) across the two groups, LMA and HMA?

2) Was there a difference between the reaction times (RT) across the two groups, LMA and HMA?

3) What differences in functional thinking were evident from EEG measures across the two groups, HMA and LMA?

4) What differences in functional thinking were evident from EEG measures across the two types of tasks: the graph-to-equation tasks and the equation-to-graph task?

\section{Theoretical Framework}

\subsection{Mathematics Anxiety}

Researchers define MA as personal feeling of fear, helplessness, and emotional instability during mathematical problem solving [9] [10] [11]. Recently, anxiety has become such a common feeling that we might consider its absence as a maladjustment [12]. MA is related to all domains of mathematics: functions, geometry, analytics, algebra etc.

Since [1] developed the original MARS instrument, MA instruments have become more specific and numerous, to measure students' MA with consideration of complex factors, including learning strategies and environment. For example, 
[13] added a Test Anxiety (TA) factor to MARS; [2] and [14] revised the instrument to work with Korean students.

Specifically, [2] examined MA using 65 items, based on the four areas of [14]: the nature of mathematics, learning strategies, test performance, and environment-all related to Korean education. In Korea, most students take private lessons on mathematics to improve their learning ability, so Ko and Yi specified MA related to learning strategies and environments that were not included in previous instruments. Ko and Yi referred to this revised instrument as the Mathematics Anxiety Scale for Students (MASS).

\subsection{Working Memory}

Working memory refers to an individual's efforts to sequence multiple mental activities or pieces of information, in ways that have not been automatized. In the traditional model of [15], this system contains three components: the central executive, a phonological loop, and a visuo-spatial sketchpad. The central executive distributes tasks to the other two components; the phonological loop handles verbal information, and the visuo-spatial sketchpad handles visual information and activities related to manipulating objects in space [16]. With regard to mathematics, the visuo-spatial sketchpad is of particular interest because of its close association with spatial reasoning.

Working memory is limited by the number of pieces of information or the number of activities that it can maintain. With regard to mental activities, [17] refer to this limitation as mental-attentional capacity, or M-capacity. This capacity to hold in mind a planned sequence of activities increases with age. Like working memory in general, it has been associated with greater mathematical ability [18].

\subsection{Brain-Based Research}

Advancement in cognitive science has introduced various brain imaging techniques. Such cognitive neuroscience techniques allow researchers to study brain functioning associated with particular experiential phenomena [19]. For example, related research on mathematics anxiety has demonstrated that higher mathematics anxiety is associated with decreased activity in the intraparietal sulcus and dorsolateral prefrontal cortex-brain regions typically associated with mathematical cognition in children [20] [21].

The researchers of [22] investigated MA during the execution of arithmetical tasks. They used EEG technology to analyze peaks in event-related potentials (ERP) among HMA participants and concluded that mathematics-anxious individuals experience difficulties in controlling for extraneous information. This experiment illuminated EEG research by [23] who had argued that children with HMA needed more time to solve addition tasks than children with LMA: "The conscious experience of anxiety seems to compete with task complexity for the limited resources of working memory" [23] [24].

The research [11] identified neurobiological mechanisms underlying mathe- 
matics anxiety using effective connectivity analysis, based on addition and subtraction problems. In a functional MRI study with 46 children (ages 7 to 9), they found that mathematics anxiety was associated with hyperactivity of the right amygdala regions that are important for processing negative emotions, as well as with the reduced activity of the posterior parietal and dorsolateral prefrontal cortex, which is involved in mathematical reasoning. Furthermore, effective connectivity between the amygdala and ventromedial prefrontal cortex regions that regulate negative emotions were elevated in children with HMA.

\subsection{Brain Research on Students' Understanding of Functions}

In this study, we focus on mathematics anxiety in the context of solving mathematical problems related to functions, which play an important role in the development of algebraic reasoning. Reasoning with functions is one of the core strands within algebraic reasoning, as identified by [25] and [26]. The research [7] suggested the complexity of the function concept arises from its many representations (e.g., graphs, equations, and tables). In our study, we chose to focus on the translation between graph and equation representations-a particularly important concept in understanding functions [27]. As such, our study resembles two previous neuroscience studies on students' understanding of functions [8] and [28], though neither of them considered the effects of MA.

The research [8] classified functions tasks into four formats: graph-to-graph, equation-to-equation, graph-to-equation and equation-to-graph. They used these formats for both linear and quadratic functions, generating a total of eight kinds of tasks for the ten students in their study-college students in New Zealand. In addition to measuring reaction times and accuracy, they measured neural activity within the neocortex of the brain through use of functional magnetic resonance imaging (fMRI). Essentially, fMRI measures neural activity by way of blood flow to areas within the brain.

The researchers found a significant increase in reaction time and a significant decrease in accuracy for the two cross-representation formats (graph-to-equation and equation-to-graph) when compared to the two same-representation formats. However, there were no such differences when comparing the two cross-representation formats, nor when comparing the two same-representation formats. Surprisingly, participants responded more accurately to tasks involving quadratic equations than to tasks involving linear equations. In a follow-up interview, all participants reported that, in general, they solved tasks in the cross-representation format by focusing on key features, such as intercept and slope.

Regarding neural activity, the researchers found, "parietal areas previously associated with number and arithmetic (the intraparietal sulcus and the posterior superior parietal lobule) were involved in the processing of mathematical functions in both graphical and algebraic formats" (p. 615) in [8]. These brain regions were even more active during cross-representations format tasks. The authors concluded that the overall neural activity during the tasks suggests "in- 
volvement of spatial and working verbal memory in the translation of functions" (p. 615) in [8].

The research [28] conducted a similar study, but with high school students in Israel. The students were divided into four groups: students identified as gifted who excelled in mathematics (G-EM), students identified as gifted who did not excel as mathematics (G-NEM), students not identified as gifted who excelled at mathematics (NG-EM), and students not identified as gifted who did not excel at mathematics (NG-NEM). Like [22], these researchers used EEG technology to analyze peaks in ERPs. The functions tasks were similar to those used by [8].

Among the four groups of students, NG-EM students exhibited the strongest ERPs and G-EM students exhibited the weakest ERPs. The researchers determined that the most prominent difference in brain activity between these two groups of students occurred within the posterior-middle electrode site, which is associated with working memory. From these results, the researchers conjectured that "problem-solving expertise developed by students without general giftedness is achieved by means of high cognitive effort" (p. 690).

\section{Methods}

\subsection{Participants}

To achieve our research purpose, we recruited 47 college students enrolled in the teacher education program of a private university located in Gyeonggi province, in South Korea. They voluntarily applied for this study through an internet announcement on their department homepages. In the analysis of data, seven students were excluded because they responded inconsistently or missed to fill all questions out.

The participants consisted of 20 students enrolled in the Department of Natural Science and 20 students enrolled in the Department of Humanities and Social Studies The researchers explained the entire process they were going to go through, assuring that all participants were comfortable with wearing the EEG cap. Participants turned in a consent form before the study, and after the study they were given a gift certificate worth about five dollars.

Each participant completed the MASS survey before beginning the EEG study. We revised words and phrases to make them more appropriate for college students, according to recommendations from educational psychologists and mathematics educators at the university where two of the researchers worked. The version of the MASS survey we used consisted of 65 items, divided into four main factors, with 12 sub factors [2]. Cronbach alpha values for the four factors proved to be between 0.7 and 0.9 .

\subsection{College Students' Performance on MASS}

The average MASS score of the 40 students in our study was 2.99 points out of 5 possible points. Based on individual scores, students were divided into the lower (LMA) and higher (HMA) mathematics anxiety groups (see Table 1). Humani- 
ties and social science students scored an average of 3.61, which was much higher than the scores of natural science students, which averaged 2.38. They scored especially high in the category of "test performance" (4.05).

The number of students for the MA groups was presented as shown Table 1. The average MASS score of the 40 students in our study was 2.99 points out of 5 possible points. Based on individual scores, students were divided into the lower (LMA) and higher (HMA) mathematics anxiety groups (see Table 1). Humanities and social science students scored an average of 3.61, which was much higher than the scores of natural science students, which averaged 2.38. They scored especially high in the category of “test performance, 4.05 ” (see Table 2).

\subsection{Reaction Time}

When an individual senses an external stimulus, this experience is assimilated to the individual's cognition by way of biological electrical signals within the central nervous system. After some degree of cognitive processing, the individual may respond. The duration of time elapsed in this span is referred to as reaction time. Reaction time may vary depending on the type of response and stimulus, as well as individual characteristics.

Table 1. Composition of students.

\begin{tabular}{cccc}
\hline & HMA & LMA & Total \\
\hline Natural Science & 5 & 15 & 20 \\
Humanities \& Social Sciences & 18 & 2 & 20 \\
Total & 23 & 17 & 40
\end{tabular}

Table 2. Students' MASS scores.

\begin{tabular}{|c|c|c|c|}
\hline Category & Factor & $\begin{array}{l}\text { Natural Science } \\
\qquad(\mathrm{SD})\end{array}$ & $\begin{array}{c}\text { Humanities and } \\
\text { Social Sciences (SD) }\end{array}$ \\
\hline \multirow{4}{*}{$\begin{array}{c}\text { Nature of } \\
\text { Mathematics }\end{array}$} & Problem Solving (I-1) & $2.38(0.94)$ & $3.65(1.09)$ \\
\hline & Mathematical Representation (I-2) & $2.3(1.20)$ & $3.35(1.09)$ \\
\hline & Mathematical Communication (I-3) & $2.17(1.11)$ & $3.23(1.13)$ \\
\hline & Abstraction (I-4) & $2.45(1.16)$ & $3.53(1.24)$ \\
\hline \multirow{3}{*}{$\begin{array}{l}\text { Learning } \\
\text { Strategy }\end{array}$} & $\begin{array}{l}\text { Learning Method and Experience } \\
\text { (II-1) }\end{array}$ & $3.14(1.25)$ & $3.81(1.05)$ \\
\hline & Self Control (II-2) & $2.53(1.14)$ & $3.39(1.29)$ \\
\hline & Motivation (II-3) & $2.33(1.13)$ & $3.05(1.43)$ \\
\hline \multirow{2}{*}{ Test Performance } & Performance (III-1) & $2.22(1.15)$ & $4.05(1.12)$ \\
\hline & Test (III-2) & $2.3(1.27)$ & $4.05(1.20)$ \\
\hline \multirow{3}{*}{ Environment } & Friends/Teachers (IV-1) & $2.37(1.08)$ & $3.83(1.22)$ \\
\hline & Teaching Method (IV-2) & $2.18(1.23)$ & $3.63(1.34)$ \\
\hline & Private Tutoring (IV-3) & $2.13(1.12)$ & $3.74(1.19)$ \\
\hline
\end{tabular}


In this study, the external stimulus was an equation or a graph presented through a program called E-prime. An equation was shown, and the participant identified a corresponding graph as correct or incorrect. Reaction time in this situation refers to the amount of time it took until the participant reached his or her own answer by pressing a key on a keyboard. In this experiment, reaction times were collected by the E-prime program, and the unit of measurement was milliseconds (ms).

\subsection{Function Tasks}

Tasks related to functions that fit the following format, with the following parameters: $y=a(x-p)^{2}+q, \quad a=\{1,-1\}, \quad p=0, \quad q=\{-2,-1,0,1,2\}$. The functions could appear as equations in the standard format, or as graphs. Participants had to determine whether a given equation fits the same function as a given graph, or vice versa (see sample task in Figure 1). Each participant solved a total of 20 graph-to-equation tasks and 20 equation-to-graph tasks.

The tasks were administered by the E-prime program in the following manner. The start of a new task was preceded by as asterisk $\left({ }^{*}\right)$, presented for $500 \mathrm{~ms}$, followed by a blank screen for $200 \mathrm{~ms}$. Then the graph or equation was presented for $3300 \mathrm{~ms}$, followed by a blank screen for $2000 \mathrm{~ms}$. Finally, the alternate format for the function (equation or graph) was presented for $3000 \mathrm{~ms}$, followed by a plus sign (+) for $500 \mathrm{~ms}$, indicating the end of the task. There was a blank screen for $500 \mathrm{~ms}$ between the presentation of tasks.

\subsection{EEG Procedures}

The researchers used a V-AMP amplifier (with electrodes and software) developed by Brain Vision. The electrode is composed of 16 channels, which can be attached to the participant's scalp underneath the cap. Professional Recorder software was used as an analyzer. Because eye movement can affect EEG measures, we used the filtering function of the analyzer to remove unrelated signals.

EEG measurements were carried out in a quiet room where the subject's body movement was minimal. Interruptions in EEG generated by the influence of electromagnetic waves of the PC and the monitor used to measure EEG were reduced by allowing a distance of about 1.5 meters between the monitor and the participant. The experiments were carried out using a shielding fiber. The researchers spent 10 minutes adjusting the electrodes for the participants to wear the EEG sensor cap, and another 10 minutes stabilizing the EEG signals. When brain waves were stable on the screen, participants were asked to raise their hand to run the E-prime program and began executing the task.

\section{Results}

\subsection{Research Question 1}

We asked whether there was a difference between the percentages of correct responses across the two groups (LMA and HMA). In the equation-to-graph 
format, the average PCR of the HMA group was $93.044 \%$ (see Table 3). Collectively, these students responded incorrectly 32 out of 460 times. Among the 32 incorrect responses, they identified 10 matching equations as not matching the graph and 22 non-matching equations as matching the graph. The average PCR of the LMA group was $92.069 \%$. Collectively, they responded incorrectly 27 out of 340 times. Among the 27 incorrect responses, they identified 9 matching equations as not matching the graph and 18 non-matching equations as matching the graph.

In the graph-to-equation format, the average PCR of the HMA group was $89.793 \%$. Collectively, they responded incorrectly 47 out of 460 times. Among the 47 incorrect responses, they identified 20 matching equations as not matching the graph and 27 non-matching equations as matching the graph. The average PCR of the LMA group was $90.699 \%$. Collectively, they responded incorrectly 32 out of 340 times. Among the 32 incorrect responses, they identified 18 matching equations as not matching the graph and 14 non-matching equations as matching the graph.

Since both anxiety groups responded differently to two different formats of tasks, to see the effect of the percent of correct answers regarding the math anxiety groups, the two-way ANOVA in which the formats of tasks were the dependent variables, was used. Table $4 \&$ Table 5 indicate that the PCA of functional tasks with two formats did not affect significantly because it assumed that the anxiety groups were the undergraduate students enrolled in the college education. In Korea, entering the college education is highly competitive, compared to other college levels. These students did not have any difficulty in solving the problems of quadratic functions.

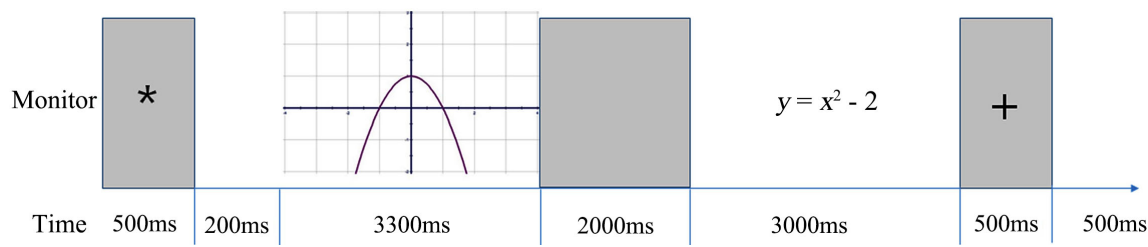

Figure 1. Sample task (graph-to-equation).

Table 3. PCR by the degree of MA in task formats.

\begin{tabular}{ccc}
\hline Groups & HMA (\%) Mean (SD) & LMA (\%) Mean (SD) \\
\hline equation to graph & $93.044(0.254)$ & $92.069(0.270)$ \\
graph to equation & $89.793(0.303)$ & $90.699(0.292)$ \\
\hline
\end{tabular}

Table 4. Between subjects factors.

\begin{tabular}{cccc}
\hline & & Value Label & N \\
\hline \multirow{2}{*}{ MA } & 1 & HMA & 46 \\
& 2 & LMA & 34 \\
\multirow{2}{*}{ Task } & 1 & E to G & 40 \\
& 2 & G to E & 40
\end{tabular}


Table 5. Tests of between subjects effects (PCR).

\begin{tabular}{cccccc}
\hline \multicolumn{5}{c}{ Dependent variable: PCR } \\
\hline Source & Type III Sum of Squares & $\mathrm{df}$ & Mean Square & $\mathrm{F}$ & Sig. \\
Corrected Model & $106.573 \mathrm{a}$ & 3 & 35.524 & 0.881 & 0.455 \\
Intercept & $647,901.841$ & 1 & $647,901.841$ & $16,073.678$ & 0.000 \\
MA & 1.841 & 1 & 1.841 & 0.046 & 0.831 \\
Task & 93.481 & 1 & 93.481 & 2.319 & 0.132 \\
MA* Task & 3.481 & 1 & 3.481 & 0.086 & 0.770 \\
Error & 3063.427 & 76 & 40.308 & & \\
Total & $665,650.000$ & 80 & & & \\
Corrected Total & 3170.000 & 79 & & & \\
\hline
\end{tabular}

a. R Squared $=0.034$ (Adjusted R Squared $=-0.005$ ).

\subsection{Research Question 2}

We asked whether there was a difference between the reaction times across the two groups (LMA and HMA). In the equation-to-graph format, the average RT of the HMA group was 1.567 seconds, and the average RT of the LMA group was 1.201 seconds. In the graph-to-equation format, the average RT of the HMA group was 2.105 seconds, and the average RT of the LMA group was 1.576 seconds (see Table 6). Generally, the HMA group responded more slowly than the LMA group.

Since the group of HMA took more time than the counterpart on both formats of tasks, to see the effect of the reaction time regarding the math anxiety groups, the two-way ANOVA in which the formats of tasks were the dependent variables, was used. Table 7 indicates that individual variables affected significantly with almost zero $\mathrm{p}$-value, at $\mathrm{p}<0.1$. This concluded that the reaction time was very important to say that the higher anxiety groups could take more time in the tasks. This coincides with the results of the research by [8].

\subsection{Research Question 3}

We asked what differences in functional thinking were evident from EEG measurement ${ }^{1}$ across the two groups: HMA and LMA. In this study, we analyzed P300-brain waves that correspond to frequencies between 200 and 400 milliseconds. P300 is considered to be an endogenous potential, as its occurrence links, not to the physical attributes of a stimulus, but to a person's reaction to it. More specifically, the P300 is thought to reflect processes involved in stimulus evaluation or categorization. We observed EEG responses during two processes. In one process, students saw an equation for a quadratic function first and memorized it, then verified whether a graph matched the equation. We named this process the equation-to-graph format, and we label it $\mathrm{F}$ format. The other process reverses the two representations, which we named the graph-to-equation format, which we label $\mathrm{G}$ format.

${ }^{1}$ Some like noises could not be perfectly controlled in the site for measuring brain waves. 
Table 6. RT by the degree of MA in task formats.

\begin{tabular}{ccc}
\hline Groups & HMA (sec) Mean $(\mathrm{SD})$ & LMA $(\mathrm{sec})$ Mean $(\mathrm{SD})$ \\
\hline equation to graph & $1.567(0.471)$ & $1.201(0.518)$ \\
graph to equation & $2.105(0.815)$ & $1.576(0.632)$ \\
\hline
\end{tabular}

Table 7. Tests of between subjects effects (RT).

\begin{tabular}{cccccc}
\hline \multicolumn{5}{c}{ Dependent Variable: RT } \\
\hline Source & Type III Sum of Squares & df & Mean Square & F & Sig. \\
\hline Corrected Model & $8.538 \mathrm{a}$ & 3 & 2.846 & 69.983 & 0.000 \\
Intercept & 202.985 & 1 & 202.985 & 4991.405 & 0.000 \\
MA & 4.010 & 1 & 4.010 & 98.603 & 0.000 \\
Task & 4.110 & 1 & 4.110 & 101.060 & 0.000 \\
MA* Task & 0.112 & 1 & 0.112 & 2.766 & 0.100 \\
Error & 3.091 & 76 & 0.041 & & \\
Total & 228.134 & 80 & & & \\
Corrected Total & 11.629 & 79 & & & \\
\hline
\end{tabular}

a. $\mathrm{R}$ Squared $=0.734$ (Adjusted R Squared $=0.724)$.

EEG measures in both the equation-to-graph format and the graph-to-equation format demonstrate that the amplitudes for the HMA group were larger than those of the LMA group. In the equation-to-graph format, the maximum amplitude of the LMA group was $28 \mu \mathrm{V}$ and the maximum amplitude of HMA group was $36 \mu \mathrm{V}$ (see Figure 2). In the graph-to-equation format, the maximum amplitude of the LMA group was $35 \mu \mathrm{V}$ and the maximum amplitude of the HMA group was $42 \mu \mathrm{V}$ (see Figure 3).

\subsection{Research Question 4}

We asked what differences in functional thinking were evident from EEG measures across the two types of tasks. Figure 4 shows that the difference between the minima of $\mathrm{F}$ and $\mathrm{G}$ formats was 11 in the LMA group. Figure 5 shows that the difference between the minima of $F$ and $G$ formats was 14 in the HMA group. The amplitude of $\mathrm{G}$ format was larger than F format in both the LMA and HMA groups.

\section{Conclusions}

MA has become a critical concern for the mathematics education community worldwide (e.g., [9] and [11]). Neuroscience methods, such as EEG, have provided mathematics education researchers with opportunities to investigate the effects of MA on mathematics performance (e.g., [22]). Our study contributes to this growing research base, as well as the broader research literature on MA, by identifying brain patterns associated with HMA as students solved tasks involving two representations of quadratic functions (graphs and equations). 


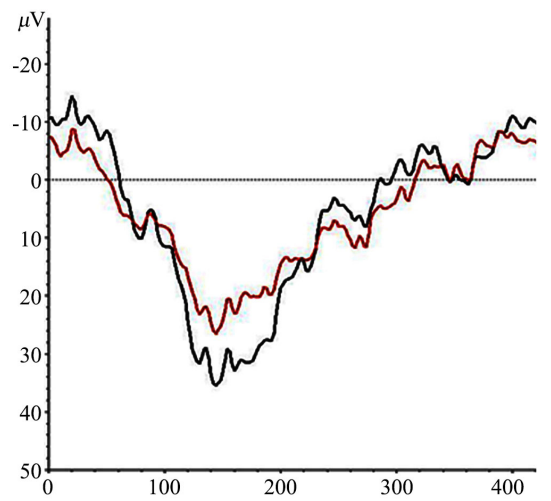

Figure 2. EEG in F format tasks (black: HMA, red: LMA).

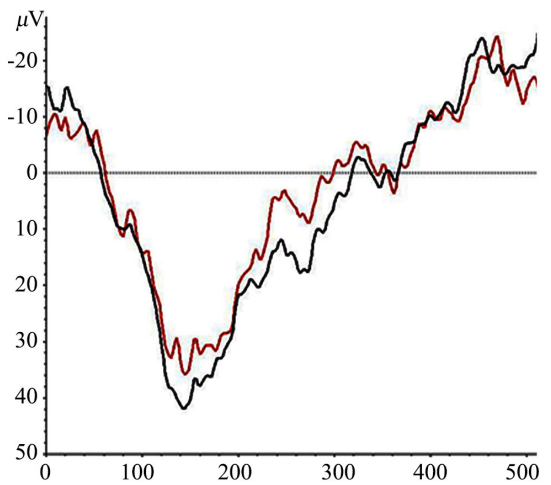

Figure 3. EEG in G format tasks (black: HMA, red: LMA).

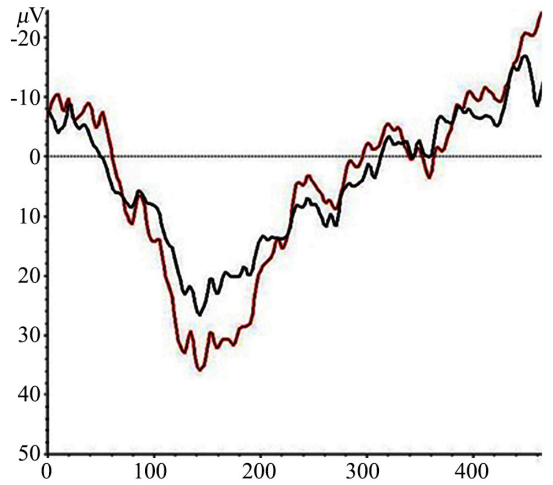

Figure 4. Comparing F with $G$ formats in LMA (black: F, red: G).

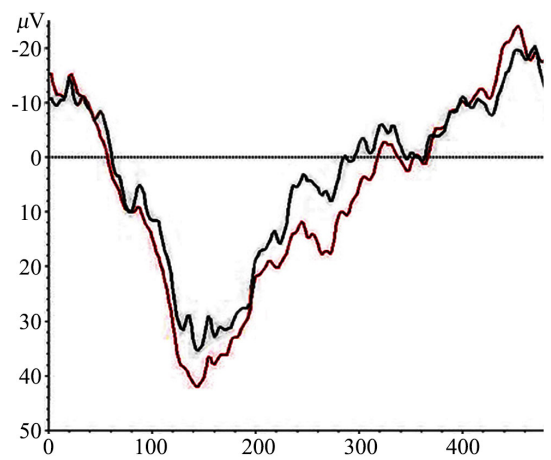

Figure 5. Comparing F with G formats in HMA (black: F, red: G). 
In addition to increased reaction times, the effects of HMA were demonstrated in the form of heightened neural activity when solving functions tasks. The research [22] found similarly heightened ERPs among young HMA students when solving arithmetic tasks. They attributed the increased amplitudes to students' difficulties in controlling for extraneous information. Collectively, these results support [24] conclusion that MA places demands on working memory, above and beyond the demands of the task itself.

Limitations in working memory are widely recognized. The research [17] offered M-capacity as a construct for modeling these limitations. Within this model, students' success on mathematical tasks depends on two factors: the cognitive demand of the task and the M-capacity of the student. If MA places additional demands on the working memory, HMA students will respond to tasks as if they were less sophisticated. There are two cases to consider within the model: either the additional demands cause the overall cognitive demand of the task to exceed a student's M-capacity, or not. In the first case, the student will fail at the task; in the second case, the student will exhibit greater use of working memory. In other words, demands of MA on limited working memory resources will make students appear younger or weaker than their peers during performance on mathematical tasks.

Comparing our results to those of [28], we find that HMA students performed in ways that were similar to NG-EM students. They successfully completed the functions tasks, but they took longer to respond and exhibited greater use of working memory. In our study, we assessed MA rather than giftedness, but HMA seemed to have the effect of making students appear less gifted, presumably because of the demands MA places on working memory. The research [11] helps explain the neurological basis for these demands. Namely, additional cognitive resources are required to regulate negative emotions associated with mathematical performance.

The research [8] found that functions tasks involving cross-representation are particularly demanding, but they found no differences in demand of accuracy between the equation-to-graph and graph-to-equation formats. In contrast, we found that the tasks in the graph-to-equation format were more cognitively demanding for our student population (as indicated by longer reaction times and higher ERP amplitudes), across both groups of students (HMA and LMA). This difference might be explained by the Korean curriculum, with which students spend more time in translating of equations to graphs. Thus, familiarity with tasks might play a role in the cognitive demand of the tasks.

HMA students can get caught in a vicious cycle of mathematical performance where poor performance on either correction rate or reaction time leads to negative emotions that characterize HMA (see Figure 6). They use valuable working memory resources managing their negative emotions during problem solving-negative emotions that are only heightened by time limits that they cannot meet with limited resources. Thus, they perform poorly on new assessments, and the cycle continues. 


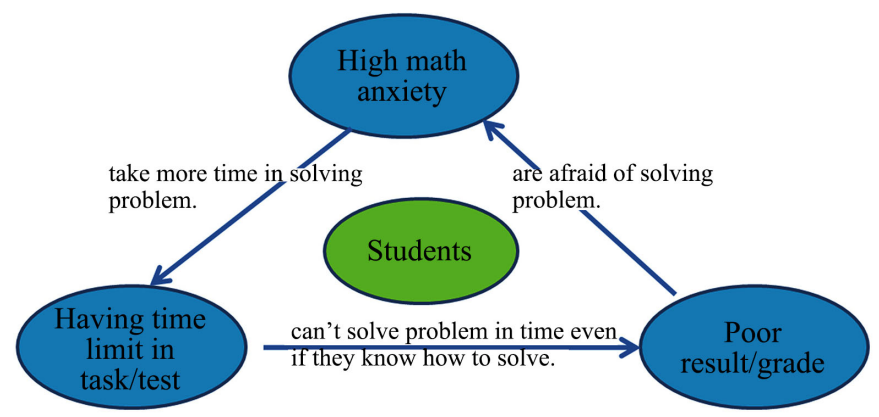

Figure 6. The vicious cycle of mathematics anxiety.

In line with previous research (e.g., [6]), we recommend that HMA students (if not all students) be provided with ample time to solve mathematical tasks, especially when the tasks are unfamiliar. Time pressure and negative emotions associated with it can compete with working memory resources. By limiting time on tasks, educators are essentially limiting opportunities for learning. Like the NG-EM students in the study by [28], many HMA students can be successful on tasks by taking advantage of extra time to work through the tasks with cognitive demands. Moreover, some of these students could be just as talented as the G-EM students, but cognitive resources appear to be sapped in controlling negative emotions associated with HMA.

\section{Conflicts of Interest}

The authors declare no conflicts of interest regarding the publication of this paper.

\section{References}

[1] Richardson, F.C. and Suinn, R.M. (1972) The Mathematics Anxiety Rating Scale: Psychometric Data. Journal of Counseling Psychology, 19, 551-554. https://doi.org/10.1037/h0033456

[2] Ko, H.K. and Yi, H.S. (2011) Development and Validation of a Mathematics Anxiety Scale for Students. Asia Pacific Education Review, 12, 509-521. https://doi.org/10.1007/s12564-011-9150-4

[3] Choi-Koh, S.S., Ko, H.K., Park, M., Han, H.S. and Hong, Y. (2005) The Assessment of Mathematics Education. Kyungmoon Co., Seoul.

[4] Choi, J.K. (2016) The Effect on Person-Centered Counselling and Petra-Mathematical Learning Method with Slow Learners. Master Thesis, Dankook University, Yongin-si.

[5] Sheffield, D. and Hunt, T. (2007) How Does Anxiety Influence Maths Performance and What Can We Do about It? MSOR Connections, 6, 19-23.

https://doi.org/10.11120/msor.2006.06040019

[6] Colome, A., Nunex-Pena, M.I. and Suarez-Pellicioni, M. (2013) Mathematical Anxiety Effects on Simple Arithmetic Processing Efficiency: An Event-Related Potential Study. Biological Psychology, 94, 517-526. https://doi.org/10.1016/j.biopsycho.2013.09.012

[7] Akkoç, H. and Tall, D. (2002) The Simplicity, Complexity and Complication of the 
Function Concept. Proceedings 26th Annual Conference of the International Group for the Psychology of Mathematics Education, Norwich, Vol. 2, 25-32.

[8] Thomas, M.O., Wilson, A.J., Corballis, M.C., Lim, V.K. and Yoon, C. (2010) Evidence from Cognitive Neuroscience for the Role of Graphical and Algebraic Representations in Understanding Function. ZDM, 42, 607-619.

https://doi.org/10.1007/s11858-010-0272-7

[9] Fennema, E. and Sherman, J. (1976) Fennema-Sherman Mathematics Attitude Scales: Instruments Designed to Measure Attitudes toward the Learning of Mathematics by Females and Males. Journal for Research in Mathematics Education, 7, 324-326. https://doi.org/10.2307/748467

[10] Tobias, S. and Weissbrod, C. (1980) Anxiety and Mathematics: An Update. Harvard Educational Review, 50, 63-70. https://doi.org/10.17763/haer.50.1.xw483257j6035084

[11] Young, C.B., Wu, S.S. and Menon, V. (2012) The Neuro-Developmental Basis of Math Anxiety. Psychological Science, 23, 492-501.

https://doi.org/10.1177/0956797611429134

[12] Andrew, S. (2003) Symptoms in the Mind. 3rd Edition, Elsevier, London.

[13] Plake, B.S. and Parker, C.S. (1982) The Development and Validation of a Revised Version of the Mathematics Anxiety Rating Scale. Educational and Psychological Measurement, 42, 551-557. https://doi.org/10.1177/001316448204200218

[14] Huh, H.J. (1996) A Study on the Factors of Mathematics Anxiety. Doctoral Dissertation, Seoul National University, Seoul.

[15] Baddeley, A. (1992) Working Memory. Science, 255, 556-559. https://doi.org/10.1126/science.1736359

[16] Logie, R.H. (1995) Visuo-Spatial Working Memory. Lawrence Erlbaum Associates, Hove.

[17] Pascual-Leone, J., Johnson, J. and Agostino, A. (2010) Mental Attention, Multiplicative Structures, and the Causal Problems of Cognitive Development. In: The Developmental Relations among Mind, Brain and Education, Springer, Berlin, 49-82. https://doi.org/10.1007/978-90-481-3666-7_3

[18] Agostino, A., Johnson, J. and Pascual-Leone, J. (2010) Executive Functions Underlying Multiplicative Reasoning: Problem Type Matters. Journal of Experimental Child Psychology, 105, 286-305. https://doi.org/10.1016/j.jecp.2009.09.006

[19] Hansen, L. and Monk, M. (2002) Brain Development, Structuring of Learning and Science Education: Where Are We Now? International Journal of Science Education, 24, 343-356. https://doi.org/10.1080/09500690110049105

[20] Ansari, D. (2008) Effects of Development and Enculturation on Number Representation in the Brain. Nature Review Neuroscience, 9, 278-291. https://doi.org/10.1038/nrn2334

[21] Rivera, S.M., Reiss, A.L., Eckert, M.A. and Menon, V. (2005) Developmental Changes in Mental Arithmetic: Evidence for Increased Functional Specialization in the Left Inferior Parietal Cortex. Cerebral Cortex, 15, 1779-1790. https://doi.org/10.1093/cercor/bhi055

[22] Suárez-Pellicioni, M., Núñez-Peña, M.I. and Colomé, À. (2013) Abnormal Error Monitoring in Math-Anxious Individuals: Evidence from Error-Related Brain Potentials. PLoS ONE, 8, e81143. https://doi.org/10.1371/journal.pone.0081143

[23] Faust, M.W. (1996) Mathematics Anxiety Effects in Simple and Complex Addition. Mathematical Cognition, 2, 25-62. https://doi.org/10.1080/135467996387534 
[24] Eysenck, M.W. and Calvo, M.G. (1992) Anxiety and Performance: The Processing Efficiency Theory. Cognition \& Emotion, 6, 409-434. https://doi.org/10.1080/02699939208409696

[25] Kaput, J. (2008) What Is Algebra? What Is Algebraic Reasoning? In: Kaput, J.J., Carraher, D.W. and Blanton, M.L., Eds., Algebra in the Early Grades, Lawrence Erlbaum Associates, New York, 5-17.

[26] National Council of Teachers of Mathematics (2000) Principles and Standards for School Mathematics. NCTM, Reston.

[27] Williams, C. (1998) Using Concept Maps to Assess Conceptual Knowledge of Function. Journal for Research in Mathematics Education, 29, 414-421. https://doi.org/10.2307/749858

[28] Waisman, I., Leikin, M., Shaul, S. and Leikin, R. (2014) Brain Activity Associated with Translation between Graphical and Symbolic Representations of Functions in Generally Gifted and Excelling in Mathematics Adolescents. International Journal of Science and Mathematics Education, 12, 669-696.

https://doi.org/10.1007/s10763-014-9513-5 